\title{
Interpretation of quantum mechanics
}

\author{
Ingvar Lindgren \\ Physics Department, Göteborg University, Göteborg, Sweden
}

(Dated: June 19, 2007)

PACS numbers:

\section{INTRODUCTION}

The nowadays most widely accepted interpretation of quantum mechanics is that of the Copenhagen school, the Copenhagen Interpretation (CI). This model, however, suffers form a number of shortcomings, the most obvious ones being:

- The measurement process plays a vital role in quantum mechanics, and in CI this requires an outside observer (classical system outside the system under investigation), which implies that the model is not applicable to a closed system, such as the entire universe.

- If the system before a measurement of a observable is in a superposition of eigenstates of the corresponding operator, the system is after a measurement transformed into one of these eigenstates. This so-called collapse or reduction of the wave function does not follow from the model but is included as a separate assumption, and it does not follow any known law of physics.

- The probability of a particular result of a measurement, the so-called Born probability law, does not follow from the model but is included as an additional postulate.

- The CI maintains an artificial border between quantum and classical systems. This dilemma can be illustrated by the famous thought experiment with the Schrödinger's cat, which when treated quantum-mechanically according to CI can be in a superposition of a dead and an alive state, until someone opens the lid and makes an inspection.

It should be noted that the dilemma with Schrödinger's cat can partly be remedied by observing that a measurement in the CI sense does not have to registered by a conscious mind. It is not even necessary to perform the measurement in order to obtain a reduction of the wave function-it is sufficient that a measurement $C A N$ be performed, as is demonstrated by "Mandel's amazing experiment" [Phys. Rev. A 47, 2293 (1993); Phys. Rev. Lett. 67, 318 (1991)]. Therefore, it is in the cat experiment not necessary to open the lid and make an observation. The signature of a measurement in CI is that it gives rise to an irreversible event. Nevertheless, the limit between the classical and the quantum worlds has to be upheld in this interpretation.

The deficiencies of CI can be eliminated by the so-called relative-state interpretation, introduced in 1957 by Hugh Everett in his PhD thesis [1] (supervisor John Wheeler [2]). The work was fairly unnoticed for more than a decade, until the model was further developed by Bryce DeWitt and others [3-5], (http://www.hedweb.com/everett/everett.html), who introduced the term Many-Words Interpretation (MWI). (For a simple introduction, see Physics Today, April 1971). The MWI term, however, is somewhat misleading, since it is not necessary to assume any "split" of the universe, and it has been rather unfortunate, since it has probably caused many people to refrain from taking the model seriously. In more recent literature the term "relative-state interpretation" is more commonly used.

During the last two decades there has been an increased activity concerning the interpretation of quantum mechanics, particularly after the discovery of the important role of the decoherence process for the basic understanding of q.m. [6-15]. A good review is found in ref. [16]. The present interest has been boosted also by the immense activity in the field of quantum information (quantum computing and quantum cryptography). (See for instance the review by Zeilinger, http://www.quantum.univie.ac.at/zeilinger/philosop.html). This development has led to a revival of 
models of the Everett type, and such models are by many considered to be the best basis for a consistent interpretation of quantum mechanics. (175 citations to the original work of Everett since year 2000 are found on the Web of Knowledge.) We shall return to these new developments in section III after a brief description of the original Everett-DeWitt model.

\section{THE EVERETT-DEWITT MANY-WORLD MODEL}

The basic idea of Everett's model is to discard the classicality of the measuring object and the collapse of the wave function and to assume that the entire world is governed by the time-dependent SE at all instances. Also other models have been proposed with this idea, such as the "modal interpretation" and the Bohm-deBroglie pilot-wave model $[16,17]$. Here we shall restrict ourselves to models of the Everett type, where presently most interest seems to be concentrated.

Following Everett, we consider a "system" $S$ to be studied and a "measuring device" $M$ with the state vectors $|S\rangle$ and $|M\rangle$, respectively. Assuming that the system initially is in a superposition of eigenstates of some observable $\alpha,|S\rangle=\sum c_{i}\left|S_{i}\right\rangle$ with $\alpha S_{i}=a_{i} S_{i}$ and the measuring device in the state $\left|M_{0}\right\rangle$, then with no interaction between them, the total state vector is the simple product

$$
\text { S } \mathbf{M}\rangle=\left(\sum c_{i}\left|S_{i}\right\rangle\right)\left|M_{0}\right\rangle
$$

When the objects are allowed to interact - according to the laws of quantum mechanics - the total state vector will be modified according to

$$
\mathbf{M}|\Psi\rangle=\sum c_{i}\left|S_{i}\right\rangle\left|M_{i}\right\rangle
$$

which implies that the states of the system and the measuring become correlated. The various components of the combined system are entangled. This corresponds to the measuring scheme devised by von Neumann and is in sharp contrast to the Copenhagen scheme, where the measuring device is assumed to be classical. The states $\left|M_{i}\right\rangle$ of the measuring device are by Everett referred to as the relative states, i.e., relative to the corresponding states of the system $S$. This interaction between entangled states has close analogy with quantum computing.

The process (2) does not represent a measurement in the traditional sense, since the state of the combined system is in a superposition of several states corresponding to different values of the quantity being measured. Everett associates each state of the measuring device with a specific observer, implying that an observer perceives only one branch of the tree - the remaining branches disappear from his view. (As we shall see below, there is not sufficient ground for this assumption in this simple model.)

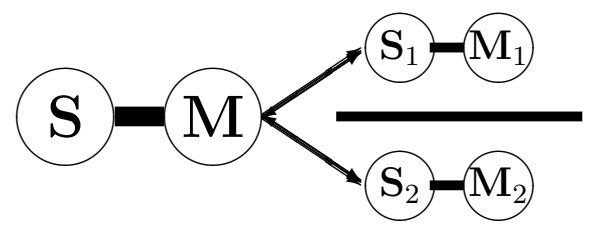

A repeated measurement of the same variable leads for the same observer to the same result. This led DeWitt to introduce the concept of "Many-World Interpretation" for this model, but we shall see that there exist other - less drastic - interpretations of this model.

There is no collapse of the wave function in this model - the Schrödinger equation (SE) is assumed to be satisfied at all instances and all places. It has also been claimed that the model leads to the Born probability law that the probability of a variable $A$ to have the value $a_{i}$ in the normalized state $|\Psi\rangle$ is

$$
P_{i}=\left|\left\langle S_{i} \mid \Psi\right\rangle\right|^{2}=\left|c_{i}\right|^{2}
$$

Later developments have shown that the model has to be modified in order to lead this result in a rigorous way.

In principle, all four difficulties of CI listed above are eliminated the by the Everett model, but it has been found necessary to include the decoherence due to the interaction with the environment in order to construct a consistent model. 


\section{LATER DEVELOPMENTS}

The decoherence is a fully quantum-mechanical process, following the time-dependent SE. The importance of this process for the interpretation of Q.M. was realized first around 1980 [6-8], and this has led to an increased activity in recent time [9-16]. The decoherence of entangled quantum systems has also been demonstrated experimentally in experiments by Haroch et al. [18] and by Zeilinger et al. [19].

There are particularly two problems with the original Everett model:

- The basis states for the process (2) are not uniquely defined

- The process for selecting one specific outcome of the measuring process is incomplete.

Concerning the first point, it can be shown that interaction with a third system is required to make the process unique

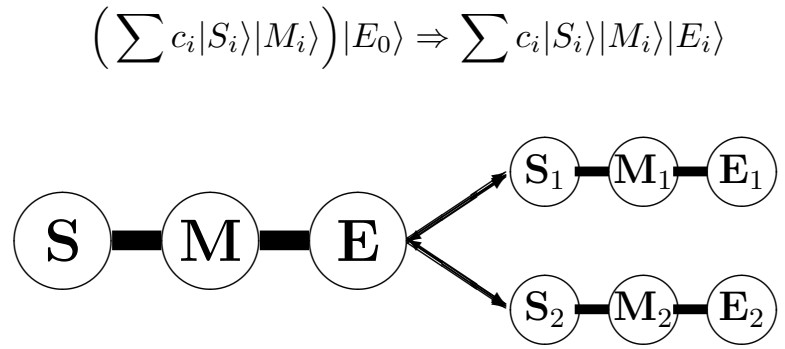

We can consider the third object as (a part of) the environment. It is then found that adding more interacting objects, will not change the basis states, which is known as the environment-induced superselection $[6,16]$. These states are also referred to as the preferred basis states or pointer states. The stability of these states implies that they will also correspond to the states in the classical limit, when a macroscopic part of the environment is included. Therefore, this model has the important property of giving a smooth transition from the quantum world to the classical world. It should be noted that this is not true for the original Everett-DeWitt model.

The interaction with the environment has the effect that the local interference (off-diagonal part of the density matrix) between the branches $S_{i}-M_{i}$ is rapidly decreasing and practically vanishing when more of the environment is included. In the limit the branches have no contact with each other, which therefore can be interpreted as the "separate worlds" in the Everett-DeWitt model.

The second problem with the Everett model concerns the specific outcome of a measurement. This problem is essentially solved with the above-mentioned environment-induced selection of basis states. We can consider an "observer" $(\mathrm{O})$ as being part of the environment [8]

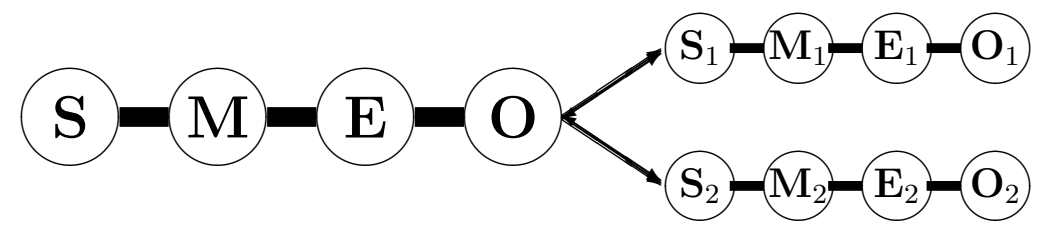

Due to the stability of the basis states, an observer perceives one definite state of the system, i.e., one specific outcome of the measurement process. For the observer this has the same effect as a collapse of the wave function. It should be observed, though, that in contrast to the CI the interaction with the environment is an entirely quantum-mechanical effect - the SE is satisfied at each instance. "The other branches are there but are not seen".

It has also be shown that the derivation of the Born statistical rule in the original Everett model is not complete. A complete proof can be obtained by including environment-induced coherence [11].

The discovery of the environment-induced superselection and of the emergence of the classical world from quantum mechanics represent the most important developments of these models in recent time. The latter part is sometimes referred to as quantum Darwinism and resolves one of the oldest disputes of traditional quantum mechanics, such as the Schrödinger's cat [9, 11] (see also the short review by Zurek 
in Wikipedia http://en.wikipedia.org/wiki/Quantum_darwinism). Many of the processes involved here are also analogous to those occurring in quantum computing [10, 14, 15].

On the more speculative side we can consider the development of the universal wave function from the beginning. Since there is no collapse in this model, the wave function will form a gigantic tree, where all branches corresponding to all events in the history preserved. At some point conscious creatures (humans) may appear, and since there is no coherence/interference between the branches, only the branch where this happens will be registered in the creature's mind - all the other branches have disappeared from the consciousness. This is essentially the anthropic principle.

\section{CONCLUSIONS}

In modern literature concerning the interpretation of quantum mechanics there is a strong tendency of abandoning the wave-function collapse of the Copenhagen interpretation and base the model entirely on the Schrödinger equation. This has led to a revival of models of Everett type, which are based upon this principle. Zurek states in his review of 2003 [9]:

The approach of Everett was to enlarge the quantum domain. Everything is now represented by a unitary evolving state vector, a gigantic superposition splitting to accommodate all the alternative consistent with the initial conditions. This is the essence of the many-worlds interpretation (MWI). It does not suffer from the dual nature of the Copenhagen interpretation.......The many-world interpretation is incomplete: it does not explain what is effectively classical and why. Nevertheless, it was a crucial conceptual breakthrough. Everett reinstated quantum mechanics as a basic tool in the search for its interpretation.

The deficiencies of the Everett model can be remedied by including decoherence into the model. Schlosshauer writes in his review from 2005 [16]:

Everett's original (1957) proposal of a relative-state interpretation of quantum mechanics has motivated several strands of interpretation.... The central idea of Everett's interpretation is to abandon [system-observer] duality and instead (i) to assume the existence of the total state $|\Psi\rangle$ representing the state of the entire universe and (ii) to uphold the universal validity of the Schrödinger equation... Decoherence adherents have typically been inclined towards relativestate interpretations... It seems natural to identify the decoherent components of the wave function with different Everett branches.

- - - -

A well-known Everett-type interpretation that relies heavily on decoherence has been proposed by Zurek [11]. This approach, termed "existential interpretation", defines the reality, or objective existence, of a state as the possibility of finding out what the state is and simultaneously leaving it unperturbed, similar to a classical state. Zurek assigns a "relative objective existence" to the robust states selected by the environment stability criterion. .... The existential interpretation continues Everett's goal of interpretation quantum mechanical using the quantum-mechanical formalism itself.

Concerning the question of the quantum-classical transition, Schlosshauer states in the same review:

The Copenhagen interpretation additionally postulates that classicality is not to be derived from quantum mechanics, for example, as the macroscopic limit of an underlying quantum structure .... In particular, the Copenhagen interpretation assumes the existence of macroscopic measurement apparatuses that obey classical physics and that are not supposed to be described in quantum-mechanical terms .... Especially in the light of the insights gained from decoherence, it seems impossible to uphold the notion of a fixed quantum-mechanical boundary on a fundamental level of theory. ... Based upon the progress already achieved by the decoherence program, it seems reasonable to anticipate that decoherence embedded in some additional interpretative structure could lead to a complete and consistent derivation of the classical world from quantum mechanics.

Zeh [13] concludes his article from 2000 in the following way:

The multi-universe interpretation (which should rather be called multi-consciousness interpretation) seems to be the only interpretation of a universal quantum theory (with an exact Schrödinger equation) that is compatible with the way the world is perceived. However, because 
of quantum non-locality it requires an appropriate modification of the traditional epistemological postulate of psycho-physical parallelism.

In this interpretation, the physical world is completely described by Everett's wave function that evolves deterministically (Laplacean). This global quantum state then defines an indeterministic (hence "branching") succession of states for all observers. Therefore, the world itself appears indeterministic-subjective in principle, but largely objective through quantum correlations (entanglement).

Actually, many of the ideas developed in recent years were introduced already in 1969 by Cooper and Van Vechten [20]. They end their article by the following conclusion:

In conclusion we claim that the process of measurement according to the interpretation given above can be placed wholly within the quantum theory. The entire system including the apparatus and even the mind of the observer can be considered to develop according to the Schrödinger equation. No discontinuous processes need to be assumed, nor is it necessary to introduce the idea of wave function reduction. The essential idea is that of the interaction of the system with a device which is irreversible in the thermodynamical sense and which eliminates as a practical matter the possibility of interference between the various branches into which the wave function is separated. This separation permits one to say that one is either on one branch or the other. The process of cognition (of being aware that something happened) is interpreted as corresponding to a certain correlation among the various branches of the wave function which allows the possibility of agreement among all observers and systems, and agreement in our own memory as to what happened. This certain correlation allows one, as a matter of convenience, to discard the other branches and renormalize the first. This, however, is only a manner of speaking since if the interaction is reversible, the possibility of interference requires the retention of all of the branches of the wave function.

This can serve as a good summary also of the more recent models developed by Zeh, Zurek and others.

\section{REFERENCES}

[1] I. H. Everett, Rev. Mod. Phys. 29, 454 (1957).

[2] J. A. Wheeler, Rev. Mod. Phys. 29, 463 (1957).

[3] B. DeWitt, Phys. Today Sept., 30 (1970).

[4] B. S. DeWitt and N. Graham, The Many-Worlds Interpretation of Quantum Mechanics (Princeton Univ. Press, Princeton, 1973).

[5] D. Deutsch, The Fabric of Reality: The Science of Parallell Universes and its implications (Penguin Books, ISBN 014027541X, 1998).

[6] W. H. Zurek, Phys. Rev. D 24, 1516 (1981).

[7] W. H. Zurek, Phys. Rev. D 26, 1862 (1982).

[8] H. D. Zeh, arXiv:quant-ph 9610014 (1996).

[9] W. H. Zurek, Rev. Mod. Phys. 75, 715 (2003).

[10] H. Oliver, D. Poulin, and W. H. Zurek, arXiv:quant-ph 0408125 (2005).

[11] W. H. Zurek, Phys. Rev. A 71, 052105 (2005).

[12] E. Joos and H. D. Z. et al., Decoherence and the Appearance of a Classical World in Quantum Theory, 2nd edition (Springer, New York, 2003).

[13] H. D. Zeh, arXiv:quant-ph 9908084 (2000).

[14] R. B.-K. H. Zurek, Phys. Rev. A 73, 062310 (2006).

[15] R. Blume-Kohout and W. H. Zurek, Fund. Phys. 35, 1857 (2005).

[16] M. Schlosshauer, Rev. Mod. Phys. 76, 1267 (2005).

[17] D. Bohm, Phil. Mag. 85, 166 (1952).

[18] M. Brune, E. Hagley, J. Dreyer, X. Maitre, A. Maali, C. Wunderlich, J. M. Raimond, and S. Haroche, Phys. Rev. Lett. 77, 4887 (1996).

[19] M. Arndt, O. Nairz, J. Vos-Andrae, C. Keler, van derZouw, and A. Zeilinger, Nature (London) 401, 680 (1999).

[20] L. N. Cooper and D. vanVechten, Am. J. Phys. 37, 1212 (1969). 